\title{
Derivadas de eigenvalores y eigenvectores en un sistema discreto
}

\section{Derivatives of Eigenvalues and Eigenvectors in a Discrete System}

José Alfredo Ramírez-Monares ${ }^{1}$

1 Technische Universität Darmstadt

\section{RESUMEN}

Un procedimiento directo para la obtención de las derivadas de los eigenvalores y eigenvectores es implementado en un sistema de dos grados de libertad. Se realiza un análisis de sensibilidad del primer eigenvalor y de los elementos del primer eigenvector con respecto a una de las rigideces como parámetro de diseño. Los resultados se presentan en gráficos de malla que permiten observar su comportamiento como funciones de dos variables.

PALABRAS CLAVE: eigenvalores; eigenvectores; análisis de sensibilidad; parámetro de diseño.

\section{ABSTRACT}

A direct procedure for obtaining the derivatives of the eigenvalues and eigenvectors is implemented in two degrees of freedom system. A sensitivity analysis of the first eigenvalue and the first eigenvector elements is performed with respect to one of the stiffness as design parameter. The results are presented in mesh graphs that allow observing their behavior as functions of two variables.

KEYWORDS: eigenvalues; eigenvectors; sensitivity analysis; design parameter.

Correspondencia:

DESTINATARIO: José Alfredo Ramírez Monares INSTITUCIÓN: Technische Universität Darmstadt (Universidad Técnica de Darmstadt)

DIRECCIÓN: Dolivostr. 15, 64293, Darmstadt, Alemania CORREO ELECTRÓNICO: ramirez@dyn.tu-darmstadt.de
Fecha de recepción: 28 de noviembre de 2020. Fecha de aceptación: 23 de febrero de 2021. Fecha de publicación: 4 de marzo de 2021.
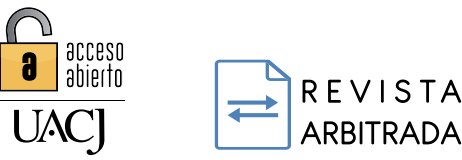

Licencia Creative Commons

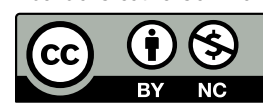




\section{INTRODUCCIÓN}

En ocasiones es deseable o necesario encontrar los efectos de un cambio de parámetro de diseño en la estabilidad y/o respuesta de un sistema mecánico. Esto ocurre, por ejemplo, cuando un diseño no es satisfactorio y la mejora se busca sobre una base clara y concreta, a veces guiada por la lógica más endeble. Incrementar o disminuir el parámetro y encontrar de nuevo la solución puede ser sensible a las dificultades numéricas inherentes, así como también costoso en tiempo de cálculo. Es deseable, entonces, tener disponible una herramienta precisa para calcular directa y eficientemente los efectos de un cambio de parámetro en un diseño. Tal herramienta tiene una amplia aplicación para encontrar el gradiente de una restricción de tipo dinámico variable en función de los parámetros de diseño en un procedimiento de optimización estructural. También es valiosa porque contribuye a la comprensión física y percepción de un problema.

En ${ }^{[1]}$ se muestran las ecuaciones para la obtención de las derivadas del eigenvalor y el eigenvector, sin embargo, no se presenta un ejemplo de aplicación de estas. En [2] se exponen las ecuaciones para la obtención solo de las derivadas de los eigenvectores en un caso numérico con una matriz característica que no es simétrica. Cardanni y Mantegazza ${ }^{[3]}$ presentan un procedimiento para la determinación de valores propios y derivadas de vectores propios que surgen en el análisis de aleteo y divergencia, enfatizando en la aplicación a los problemas de ambos aspectos, ya que la idea básica del método unifica la determinación de una solución y de sus derivadas en un enfoque único. Murthy y Haftka ${ }^{[4]}$, Lin y $\mathrm{Ng}^{[5]}$ y Yong [6] presentan un amplio estudio de algunos métodos para el análisis de sensibilidad del problema de valores propios algebraicos para matrices no hermitianas. Ahí se establecen pautas generales para la selección del método más eficiente y se presentan casos numéricos, pero no se muestran gráficos de superficie donde se permita observar los cambios con respecto a dos variables.

En el presente trabajo se utiliza la aproximación directa mostrada en ${ }^{[7]}$ y ${ }^{[8]}$ para la obtención de las derivadas de los eigenvalores y eigenvectores. Las soluciones se representan en gráficos de malla que permiten observar el comportamiento de los valores con respecto a cambios en dos parámetros de rigidez $k_{1}$ y $k_{2}$.

Esta representación permite una mejor percepción física y comprensión del problema.

\section{METODOLOGÍA}

La metodología del presente trabajo de investigación consiste en:

1. Definición del problema del eigenvalor para sistemas mecánicos discretos. Solución del problema del eigenvalor y eigenvectores a partir de condición de normalización de la matriz de masa.

2. Desarrollo de las ecuaciones que definen las derivadas de la frecuencia y eigenvectores con respecto a un parámetro de diseño.

3. Obtención de los gráficos de malla.

4. Análisis de los resultados y conclusiones.

A continuación se muestra el desarrollo de las ecuaciones del presente trabajo de investigación.

\section{A. DERIVADAS DE LA FRECUENCIA Y EIGENVECTORES EN SISTEMAS ESTRUCTURALES DISCRETOS}

El análisis de frecuencia natural para el caso de vibración libre no amortiguada de una estructura discretizada consiste en encontrar una solución al conjunto homogéneo de matrices de orden $n$.

$$
\left\{[K]-\omega_{j}^{2}[M]\right\}\left\{\psi_{j}\right\}=0 \quad j=1,2, \ldots, \mathrm{n}
$$

donde $[K]$ es la matriz de rigidez, $[M]=\left[M_{s}\right]+[\bar{M}],\left[M_{s}\right]$ y $[\bar{M}]$ son las matrices de masa estructural y no estructural, respectivamente. El eigenvalor $\omega_{j}^{2}$ es la frecuencia circular asociada con el $j$-ésimo modo de vibración. El eigenvector $\left\{\psi_{j}\right\}$ es normalizado frecuentemente con la matriz de masa, como se muestra en la Ecuación (2).

$$
\left\{\psi_{j}\right\}^{T}[M]\left\{\psi_{j}\right\}=1
$$

El gradiente de la frecuencia circular con respecto a la variable de diseño $x_{i}$ se puede obtener diferenciando la Ecuación (1), esto es:

$$
\begin{array}{r}
\left([K]-\omega_{j}^{2}[M]\right) \frac{d\left\{\psi_{j}\right\}}{d x_{i}}-\frac{d \omega^{2}}{d x_{i}}[M]\left\{\psi_{j}\right\} \\
=-\left(\frac{d[K]}{d x_{i}}-\omega_{j}^{2} \frac{d[M]}{d x_{i}}\right)\left\{\psi_{j}\right\} \\
\frac{d \omega^{2}}{d x_{i}}=\frac{\left\{\psi_{j}\right\}^{T}\left(\frac{d[K]}{d x_{i}}-\omega_{j}^{2} \frac{d[M]}{d x_{i}}\right)\left\{\psi_{j}\right\}}{\left\{\psi_{j}\right\}^{T}[M]\left\{\psi_{j}\right\}}
\end{array}
$$


donde $\left\{\psi_{j}\right\}$ es el componente del modo de vibración asociado con el $i$-ésimo elemento. El gradiente del eigenvector se obtiene diferenciando la Ecuación (2), lo que resulta en lo siguiente:

$$
\left\{\psi_{j}\right\}^{T}[M] \frac{d\left\{\psi_{j}\right\}}{d x_{i}}=\frac{1}{2}\left\{\psi_{j}\right\}^{T} \frac{d[M]}{d x_{i}}\left\{\psi_{j}\right\}
$$

Para la obtención de las derivadas de los eigenvalores y eigenvectores se puede usar la aproximación directa, la cual combina las Ecuaciones (4) y (5):

$$
\begin{aligned}
& {\left[\begin{array}{cc}
{[K]-\omega_{j}^{2}[M]} & -[M]\left\{\psi_{j}\right\} \\
-\left\{\psi_{j}\right\}^{T} & 0
\end{array}\right]\left\{\begin{array}{c}
\frac{d\left\{\psi_{j}\right\}}{d x_{i}} \\
\frac{d \omega^{2}}{d x_{i}}
\end{array}\right\}} \\
& =\left\{\begin{array}{c}
-\left(\frac{d[K]}{d x_{i}}-\omega_{j}^{2} \frac{d[M]}{d x_{i}}\right)\left\{\psi_{j}\right\} \\
\frac{1}{2}\left\{\psi_{j}\right\}^{T} \frac{d[M]}{d x_{i}}\left\{\psi_{j}\right\}
\end{array}\right\}
\end{aligned}
$$

\section{B. MODELO DE DOS MASAS Y TRES RESORTES}

La Figura 1 muestra el sistema mecánico presentado en Haftka y Gürdal [7]. Es un sistema discreto con tres resortes de rigideces $k_{1}, k_{2}$ y $k_{3}$, dos grados de libertad $u_{1}$ y $u_{2}$, que son los desplazamientos horizontales de las masas $m_{1}$ y $m_{2}$.

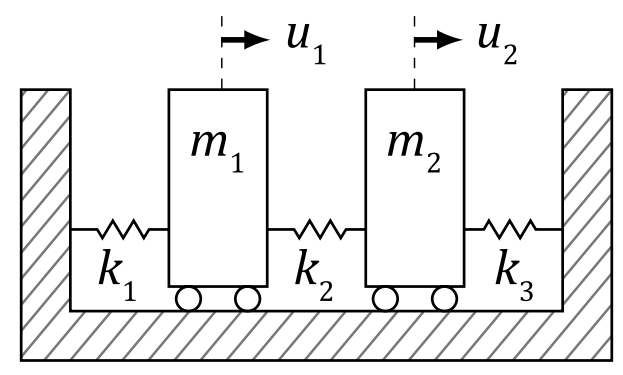

Figura 1. Sistema mecánico discreto de dos masas y tres resortes.

Las matrices de masa y rigidez se muestran a continuación.

$$
\begin{gathered}
{[M]=\left[\begin{array}{cc}
m_{1} & 0 \\
0 & m_{2}
\end{array}\right]} \\
{[K]=\left[\begin{array}{cc}
k_{1}+k_{2} & -k_{2} \\
-k_{2} & k_{2}+k_{3}
\end{array}\right]}
\end{gathered}
$$

$$
\begin{array}{lc}
{\left[\begin{array}{cc}
k_{1}+k_{2}-\omega^{2} m_{1} & -k_{2} \\
-k_{2} & k_{2}+k_{3}-\omega^{2} m_{2}
\end{array}\right]\left\{\begin{array}{l}
\psi_{1} \\
\psi_{2}
\end{array}\right\}_{j}} & =\left\{\begin{array}{l}
0 \\
0
\end{array}\right\}
\end{array}
$$

Igualando a cero el determinante se obtienen las frecuencias:

$$
\begin{aligned}
& \omega_{1}^{2}=\frac{1}{2}\left[k_{1}+2 k_{2}-k_{3}-\sqrt{4 k_{2}^{2}+\left(k_{1}-k_{3}\right)^{2}}\right] \\
& \omega_{2}^{2}=\frac{1}{2}\left[k_{1}+2 k_{2}-k_{3}-\sqrt{4 k_{2}^{2}+\left(k_{1}-k_{3}\right)^{2}}\right] \\
& \frac{-k_{1}+k_{3}+\sqrt{4 k_{2}^{2}\left(k_{1}-k_{3}\right)^{2}}}{2 k_{2}}\left\{\psi_{1}\right\}_{1}+\left\{\psi_{2}\right\}_{1}=0 \\
& \frac{-k_{1}+k_{3}-\sqrt{4 k_{2}^{2}\left(k_{1}-k_{3}\right)^{2}}}{2 k_{2}}\left\{\psi_{1}\right\}_{2}+\left\{\psi_{2}\right\}_{2}=0
\end{aligned}
$$

Ahora, haciendo uso de la condición de normalización mostrada en la Ecuación (2), la ecuación adicional para el primer eigenvector resulta:

$$
\left\{\psi_{1}\right\}_{1}^{2}+\left\{\psi_{2}\right\}_{1}^{2}=1
$$

El primer eigenvector se encuentra al solucionar los sistemas (12) y (14). Entonces, este se puede utilizar para obtener las derivadas de las frecuencias y los eigenvectores.

\section{RESULTADOS}

Todos los resultados aquí mostrados son obtenidos utilizando $m_{1}=m_{1}=m_{1}=1 \mathrm{~kg}, k_{3}=1 \mathrm{~N} / \mathrm{m}$ y las variaciones en las rigideces $k_{1}=0,0.1,0.2, \ldots, 1$ y $k_{2}=0.1,0.2$, $0.3, \ldots, 1$. Se obtienen las derivadas de la frecuencia y de los elementos del primer eigenvector con respecto al parámetro de diseño: la rigidez $k_{1}$.

En la Figura 2 se muestra un gráfico de malla con los valores de los primeros eigenvalores correspondientes a la frecuencia menor, como función de las rigideces $k_{1} \mathrm{y}$ $k_{2}$. Ahí se observa que este primer eigenvalor crece con pendiente positiva respecto a valores crecientes de $k_{1}$. También se observa que los valores se mantienen constantes en $k_{1}=1$. 


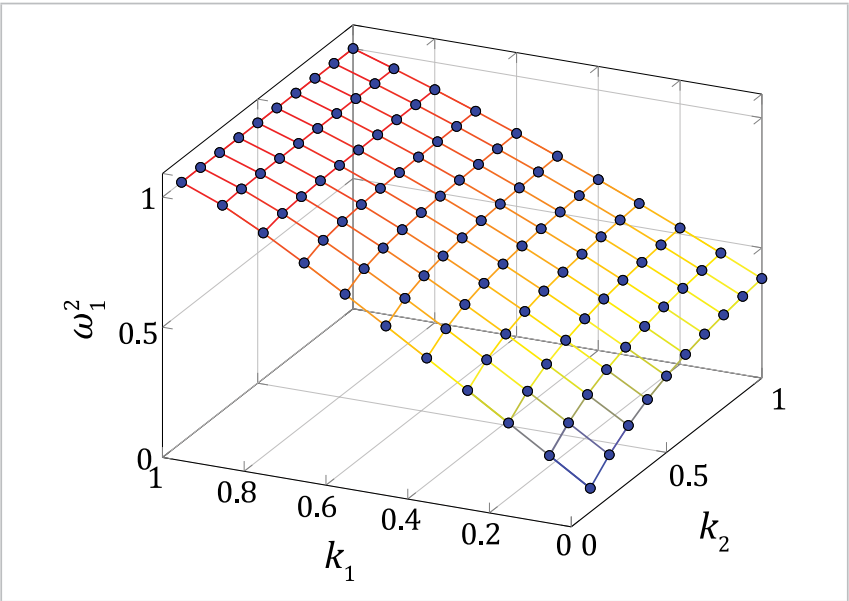

Figura 2. Eigenvalores $\omega_{1}^{2}$ para distintos valores de $k_{1}$ y $k_{2}$.

En la Figura 3 se muestran las derivadas de la primera frecuencia con respecto a la rigidez $k_{1}$ para distintas rigideces $k_{1}$ y $k_{2}$. Se observa que las derivadas tienden a un valor constante de 0.5 para valores que se aproximan a $k_{1}=1$; la tasa de cambio crece para ambas rigideces a medida que se aproxima a cero.

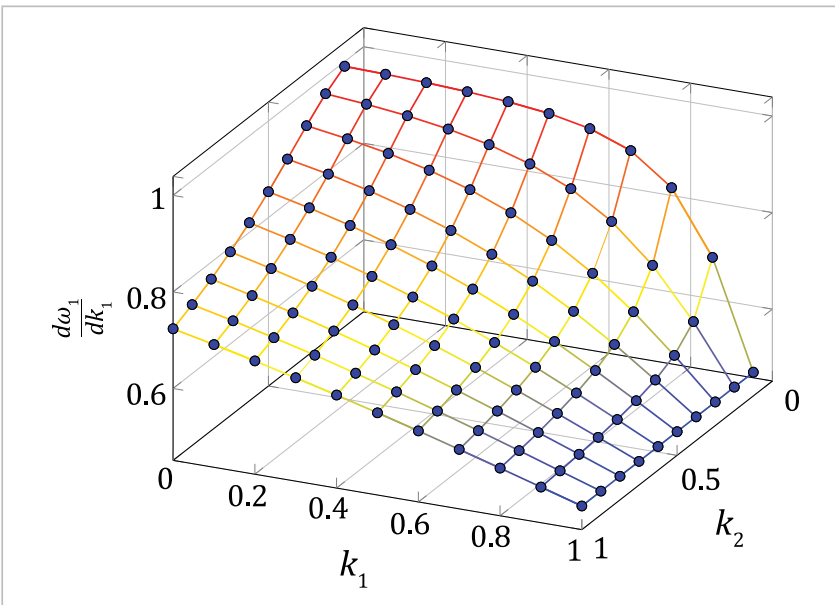

Figura 3. Derivadas del eigenvalor con respecto a cambios en la rigidez $\frac{d \omega_{1}}{d k_{1}}$ para distintos valores de $k_{1}$ y $k_{2}$.

En la Figura 4 se muestran los valores del primer elemento del eigenvector correspondiente a la frecuencia menor para distintas rigideces $k_{1}$ y $k_{2}$. Nuevamente se observa una tendencia hacia un valor constante de 0.7 para valores que se aproximan a $k_{1}=1$. Los valores aumentan para ambas rigideces a medida que se aproxima a cero.

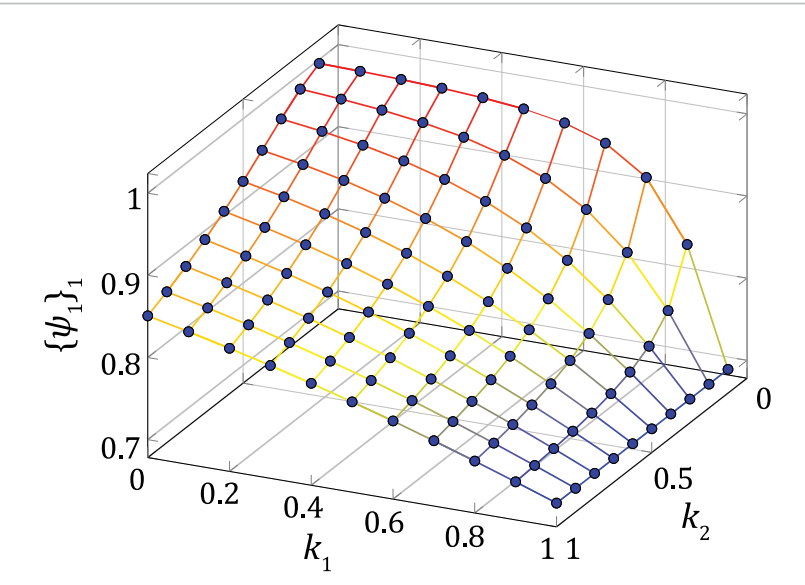

Figura 4. Primer elemento del eigenvector $\left\{\psi_{1}\right\}_{1}$ para distintos valores de $k_{1}$ y $k_{2}$.

En la Figura 5 se muestran las derivadas del primer elemento del eigenvector con respecto a la rigidez $k_{1}$ para distintas rigideces $k_{1}$ y $k_{2}$. Se observa que las derivadas tienen un valor que se mantiene cuasi constante en cero en todo el plano, excepto cuando se acerca hacia la esquina $k_{1}=1$ y $k_{2}=0$, donde se advierte un descenso drástico.

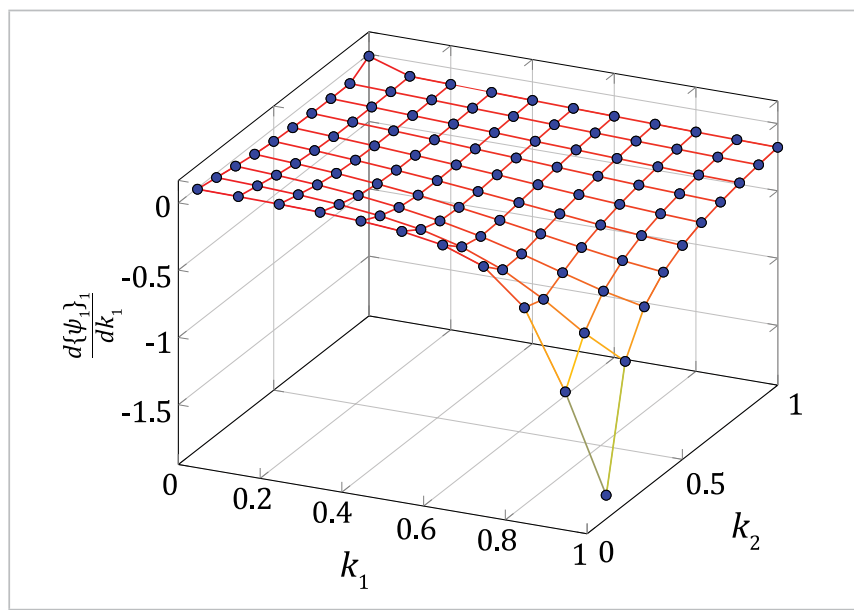

Figura 5. Derivadas del primer elemento del eigenvector con respecto a cambios en la rigidez $\frac{d\left\{\psi_{1}\right\}_{1}}{d k_{1}}$ para distintos valores de $k_{1} \mathrm{y} k_{2}$.

En la Figura 6 se muestran los valores del segundo elemento del eigenvector correspondiente a la frecuencia menor para distintas rigideces $k_{1}$ y $k_{2}$. Nuevamente se observa una tendencia hacia un valor constante de 0.7 para valores que se aproximan a $k_{1}=1$. Los valores disminuyen para ambas rigideces a medida que se aproxima a cero. 


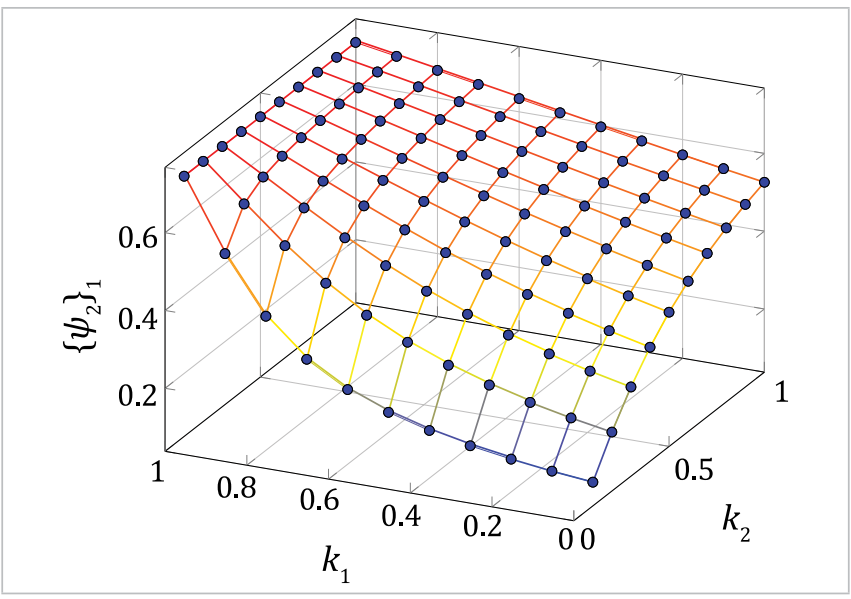

Figura 6. Segundo elemento del eigenvector $\left\{\psi_{2}\right\}_{1}$ para distintos valores de $k_{1}$ y $k_{2}$.

En la Figura 7 se muestran las derivadas del segundo elemento del eigenvector con respecto a la rigidez $k_{1}$ para distintas rigideces $k_{1}$ y $k_{2}$. Se observa que las derivadas tienen un valor que crece drásticamente cuando se acerca hacia la esquina $k_{1}=1$ y $k_{2}=0$.

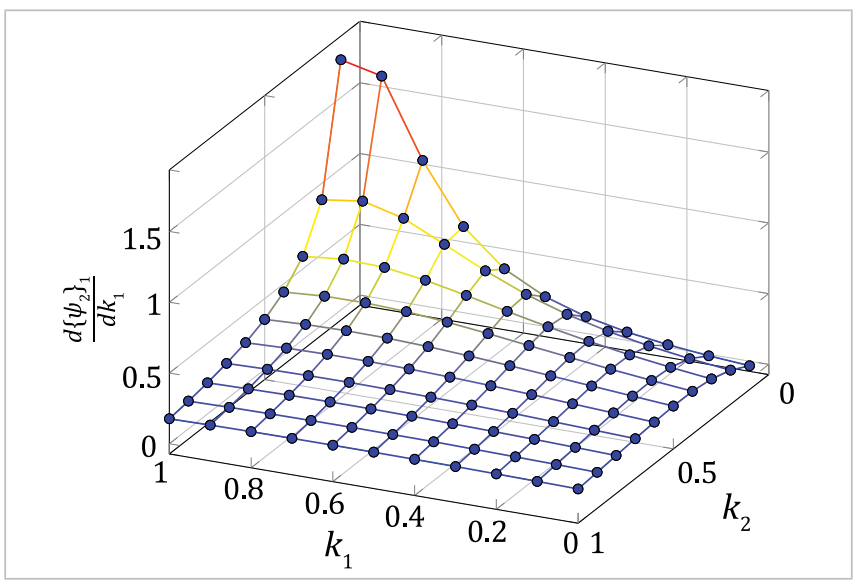

Figura 7. Derivadas del segundo elemento del eigenvector con respecto a cambios en la rigidez $\frac{d\left\{\psi_{2}\right\}_{1}}{d k_{1}}$ para distintos valores de $k_{1} \mathrm{y} k_{2}$.

Para $k_{1}=k_{2}=k_{1}=k_{3}=1$ el valor de la frecuencia fundamental es $\omega_{1}^{2}=1$. Los valores permanecen constantes para $k_{1}=1$ en las Figura 2, 4 y 6 . Esto se debe a que al ser iguales las rigideces $k_{1}$ y $k_{3}$, el sistema tiene una simetría que produce frecuencias dobles asociadas a este par, independientemente de los valores de $k_{2}$. Esta simetría no se produce con valores $k_{2}=k_{3}$ ni con $k_{1}=k_{2}$.

En general puede observarse que la primera frecuencia es muy sensible a cambios en la rigidez $k_{1} \mathrm{y}$ tal variación es dependiente de las demás rigideces.
Acerca de los elementos del eigenvector $\left\{\psi_{1}\right\}_{1}$ y $\left\{\psi_{2}\right\}_{1}$, en los gráficos se observa que si se desea una primera forma modal con un desplazamiento menor en el grado de libertad $u_{1}$, esto se consigue en los valores de rigidez $k_{1}=1$, produciendo a su vez un desplazamiento máximo en el grado de libertad $u_{2}$.

\section{CONCLUSIONES}

Se presentaron las ecuaciones para obtener las frecuencias de vibración a partir de la formulación del problema del eigenvalor en un sistema discreto con dos grados de libertad.

Se obtuvieron los gráficos de malla de la primera frecuencia, sus correspondientes elementos del eigenvector y las derivadas de todos estos con respecto a la variable de diseño $k_{1}$. Se observó cómo la frecuencia doble correspondiente a la igualdad en las rigideces $k_{1}=k_{3}$ anula el efecto de la rigidez $k_{2}$ en la primera frecuencia.

Se demostró también la relación entre las rigideces y los valores para obtener desplazamientos máximos y mínimos en cada grado de libertad en la forma modal de la primera frecuencia.

\section{REFERENCIAS}

[1] L. C. Rogers, "Derivatives of Eigenvalues and Eigenvectors", AIAA J., vol. 8 no. 5, 1970. DOI: 10.2514/3.5795.

[2] R. B. Nelson, "Simplified Calculation of Eigenvector Derivatives", AIAA J., vol. 14, no. 9, 1976. DOI: 10.2514/3.7211.

[3] C. Cardani y P. Mantegazza, "Calculation of Eigenvalue and Eigenvector Derivatives for Algebraic Flutter and Divergence Eigenproblems", AIAA J., vol. 17, no. 4, 1978. DOI: $10.2514 / 3.61140$.

[4] D. V. Murthy y R. T. Haftka, "Derivatives of Eigenvalues and Eigenvectors of a General Complex Matrix", Int. J. Numer. Methods Eng., vol. 26, no. 2, pp. 293-311, 1988. DOI: 1620260202.

[5] R. M. Lin y T. Y. Ng, "Eigenvalue and eigenvector derivatives of fractional vibration systems", Mech. Syst. Signal Process., vol. 127, pp. 423-440, jul. 2019. DOI: 10.1016/j. ymssp.2019.03.014. 
[6] Y. Xia, S. Weng, Y. L. Xu y H. P. Zhu, “Calculation of eigenvalue and eigenvector derivatives with the improved Kron's substructuring method", Struct. Eng. Mech., vol. 36, no. 1, pp. 37-55, 2010. DOI: 10.12989/ sem.2010.36.1.037.

[7] R. T. Haftka and Z. Gürdal, Elements of Structural Optimization, 3. a ed. Dordrecht, The Netherlands: Kluwer Academic Publishers, 1991.
[8] K. T. Abou-Moustafa, “On Derivatives of Eigenvalues and Eigenvectors of the Generalized Eigenvalue Problem", Montreal, QC, Canada, MTR No. TR-CIM-10-09, 2010. Consultado: 9 de diciembre de 2020. [En línea]. Disponible en: http://aboumoustafa.org/KAM_MCGILLTR2009.pdf. 